Table 1. Spearman Rho Correlation between timed function tests and MMT8, FI-2, patient and physician VAS

\begin{tabular}{|c|c|c|c|}
\hline & $\Delta 30$ s rise from chair test & 30 s arm lift test $\Delta$ & 2 min walk test \\
\hline $\begin{array}{l}\Delta \text { MMT8 } \\
\Delta \text { FI-2 }\end{array}$ & 0.382 & 0.337 & $0.724^{\star *}$ \\
\hline Hip flexion right & 0.388 & 0.413 & 0.314 \\
\hline Hip flexion Left & $0.503^{*}$ & 0.416 & 0.422 \\
\hline Neck flexion & $0.600^{* *}$ & $0.590^{* *}$ & $0.610^{* *}$ \\
\hline Shoulder flexion right & 0.183 & 0.300 & 0.239 \\
\hline Shoulder flexion left & 0.393 & 0.222 & 0.207 \\
\hline Shoulder abduction right & 0.236 & 0.222 & 0.348 \\
\hline Shoulder abduction left & 0.182 & 0.236 & 0.273 \\
\hline Step test right & $0.744^{\star \star}$ & $0.489^{*}$ & 0.326 \\
\hline Step test left & 0.840 & $0.500^{*}$ & 0.378 \\
\hline Heel rise & 0.442 & 0.294 & 0.388 \\
\hline Toe rise & 0.446 & 0.291 & 0.419 \\
\hline$\Delta$ Physician VAS & $-0.508^{*}$ & $-0.506^{\star \star}$ & -0.215 \\
\hline$\Delta$ Patient VAS & $-0.600^{* *}$ & $-0.597^{\star \star}$ & -0.249 \\
\hline
\end{tabular}

$\Delta \rightarrow$ change from baseline to 3 months ${ }^{\star} \rightarrow$ Correlation is significant at the 0.05 level ${ }^{* *} \rightarrow$ Correlation is significant at the 0.01 level

Conclusion: Timed function tests correlated well with MMT 8 and parameters with in FI-2. Thus these tests are good alternatives in assessing disease activity and response assessment in inflammatory myositis.

References:

[1] Selva-O'Callaghan A, Pinal-Fernandez I, Trallero-Araguás E, Milisenda JC, Grau-Junyent JM, Mammen AL. Classification and management of adult inflammatory myopathies. Lancet Neurol. 2018; 17:816-28.

[2] Rider LG, Aggarwal R, Machado PM, Hogrel J-Y, Reed AM, Christopher-Stine $\mathrm{L}$, et al. Update on outcome assessment in myositis. Nat. Rev. Rheumatol. 2018;14:303-18.

[3] Alexanderson H, Broman L, TollbÄck A, Josefson A, Lundberg IE, StenstrÖm $\mathrm{CH}$. Functional index-2: Validity and reliability of a disease-specific measure of impairment in patients with polymyositis and dermatomyositis. Arthritis Rheum. 2006;55:114-22.

Disclosure of Interests: : None declared

DOI: 10.1136/annrheumdis-2020-eular.6078

\section{SAT0315 INHIBITION OF MICROSOMAL PROSTAGLANDIN E SYNTHASE-1 (MPGES-1) BY GS-248 REDUCES PROSTAGLANDIN E2 BIOSYNTHESIS WHILE INCREASING PROSTACYCLIN IN HUMAN SUBJECTS}

C. Edenius $^{1,2}$, G. Ekström ${ }^{1,3}$, J. Kolmert ${ }^{3}$, R. Morgenstern ${ }^{1,3}$, P. Stenberg ${ }^{1,3}$, P. J. Jakobsson ${ }^{1,4,5}$, G. Tornling ${ }^{1,6}$. 'Gesynta Pharma, Stockholm, Sweden; ${ }^{2}$ Karolinska Institutet, Department of Medicine Solna, Stockholm, Sweden; ${ }^{3}$ Karolinska Institutet, The Institute of Environmental Medicine, Stockholm, Sweden; ${ }^{4}$ Karolinska University Hospital, Stockholm, Sweden; ${ }^{5}$ Karolinska Institutet, Department of Medicine Solna, Rheumatology Unit, Stockholm, Sweden; ${ }^{6}$ Karolinska Institutet, Department of Medicine Solna, Respiratory Unit, Stockholm, Sweden

Background: Microsomal prostaglandin E synthase-1 (mPGES-1) catalyzes the formation prostaglandin ( $P G) E_{2}$ from cyclooxygenase derived $\mathrm{PGH}_{2}{ }^{(1,2)}$. Inhibition of $\mathrm{mPGES}-1$ leads to reduction of pro-inflammatory $\mathrm{PGE}_{2}$, while in vessels there is a concomitant increase of vasoprotective prostacyclin $\left(\mathrm{PGI}_{2}\right)$ via shunting of $\mathrm{PGH}_{2}{ }^{(3,4)}$. Apart from relieving symptoms in experimental animal models of inflammation, inhibitors of mPGES-1 cause relaxation of human medium sized arteries $^{(4)}$ and resistance arteries ${ }^{(5)}$. The prostaglandin profile following $\mathrm{mPGES}-1$ inhibition, explains the anti-inflammatory effects and also opens for the possibility of treating inflammatory diseases with concomitant vasculopathies. GS-248 is a potent and selective inhibitor of $\mathrm{mPGES}-1$ exhibiting sub-nanomolar $\mathrm{IC}_{50}$ in human whole blood ex vivo.

Objectives: To evaluate safety, tolerability, pharmacokinetics and pharmacodynamics of GS-248.

Methods: Healthy males and females (age 18-73 years) were included in the study. Six cohorts were administrated single oral doses of 1-300mg GS-248 $(n=36)$ or placebo $(n=12)$, three cohorts were administered once daily doses of 20-180mg GS-248 ( $n=18)$ or placebo $(n=12)$ over ten days. In addition, 8 subjects were treated in a separate cohort with $200 \mathrm{mg}$ celecoxib bid for ten days. Blood samples were drawn for measurement of GS-248 exposure and production of $\mathrm{PGE}_{2}$ after LPS incubation ex vivo. The content of $\mathrm{PGE}_{2}$ and $\mathrm{PGI}_{2}$ metabolites was measured in urine. All analyses were performed by LC-MS/MS.

Results: GS-248 was safe and well tolerated at all tested dose levels. Maximum plasma concentration was achieved $1-2.5$ hours after dosing, and half-life was about 10 hours. Induced PGE formation ex vivo, catalyzed by mPGES-1, was completely inhibited for 24 hours after a single low dose (40mg) of GS-248. In urine, GS-248 dose-dependently reduced the excretion of $\mathrm{PGE}_{2}$ metabolite by more than $50 \%$ whereas the excretion of $\mathrm{PGI}_{2}$ metabolite increased more than twice the baseline levels. In the celecoxib cohort urinary metabolites of both $\mathrm{PGE}_{2}$ and $\mathrm{PGI}$, were reduced with approx $50 \%$.

Conclusion: GS-248 at investigated oral doses was safe and well tolerated. There was a sustained inhibition of LPS induced PGE formation in whole blood. In urine, there was a metabolite shift showing reduced $P G E_{2}$ and increased $P G I_{2}$ while celecoxib reduced both $\mathrm{PGE}_{2}$ and $\mathrm{PGI}$ metabolites. This suggests that selective inhibition of mPGES-1 results in systemic shunting of $\mathrm{PGH}_{2}$ to $\mathrm{PGI}$ formation, leading to anti-inflammatory and vasodilatory effects, while preventing platelet activation. The results warrant further evaluation of GS-248 in inflammatory conditions with vasculopathies such as Digital Ulcers and Raynaud's Phenomenon in Systemic Sclerosis.

\section{References:}

[1] Korotkova M, Jakobsson PJ. Persisting eicosanoid pathways in rheumatic diseases. Nat Rev Rheumatol. 2014;10:229-41

[2] Bergqvist F, Morgenstern R, Jakobsson PJ. A review on mPGES-1 inhibitors: From preclinical studies to clinical applications. Prostaglandins Other Lipid Mediat. 2019;147:106383

[3] Kirkby NS, et al. Mechanistic definition of the cardiovascular mPGES-1/ COX-2/ADMA axis. Cardiovasc Res. 2020

[4] Ozen G, et al. Inhibition of microsomal PGE synthase-1 reduces human vascular tone by increasing PGI2: a safer alternative to COX-2 inhibition. $\mathrm{Br} J$ Pharmacol. 2017; 174:4087-98

[5] Larsson K, et al. Biological characterization of new inhibitors of microsomal $P G E$ synthase-1 in preclinical models of inflammation and vascular tone. $\mathrm{Br}$ J Pharmacol. 2019;176:4625-38

Disclosure of Interests: : Charlotte Edenius Shareholder of: Gesynta Pharma Consultant of: Gesynta Pharma,, Gunilla Ekström Shareholder of: Gesynta Pharma, Consultant of: Gesynta Pharma,, Johan Kolmert Consultant of: Gesynta Pharma,, Ralf Morgenstern Shareholder of: Gesynta Pharma, Employee of Gesynta Pharma, Patric Stenberg Shareholder of: Gesynta Pharma, Employee of: Gesynta Pharma, Per-Johan Jakobsson Shareholder of: Gesynta Pharma Grant/research support from: Gesynta Pharma, AstraZeneca,, Göran Tornling Shareholder of: Gesynta Pharma, Vicore Pharma,, Consultant of: Gesynta Pharma, Vicore Pharma, AnaMar DOI: 10.1136/annrheumdis-2020-eular.5503

\section{SAT0316 ANTI-PM/SCL ANTIBODIES IN SYSTEMIC SCLEROSIS: CLINICAL ASSOCIATIONS IN THE RESCLE COHORT}

N. Iniesta-Arandia ${ }^{1}$, G. Espinosa ${ }^{2}$, A. Guillen del Castillo ${ }^{3}$, C. Tolosa ${ }^{4}$, G. M. Lledó ${ }^{1}$, D. Colunga Argüelles ${ }^{5}$, C. González-Echávarri ${ }^{6}$, L. Sáez-Comet ${ }^{7}$, N. Ortego ${ }^{8}$, J. A. Vargas-Hitos ${ }^{9}$, M. Rubio-Rivas ${ }^{10}$, M. Freire ${ }^{11}$, J. J. Rios ${ }^{12}$ M. Rodriguez-Carballeira ${ }^{13}$, L. Trapiella Martínez ${ }^{14}$, V. Fonollosa $\mathrm{Pla}^{3}$, C. P. Simeón-Aznar ${ }^{3}$, O. B. O. R. I. Autoimmune Diseases Study Group (Geas) ${ }^{15}$. ${ }^{1}$ Hospital Clinic, Barcelona, Spain; ${ }^{2}$ Hospital Clinic, Autoimmune Diseases, Barcelona, Spain; ${ }^{3}$ Hospital Vall d'Hebron, Barcelona, Spain; ${ }^{4}$ Hospital Parc Taulí, Sabadell, Spain; ${ }^{5}$ Hospital Universitario Central de Asturias, Oviedo, Spain; ${ }^{6}$ Hospital de Cruces, Barakaldo, Spain; ${ }^{7}$ Hospital Universitario Miguel Servet, Zaragoza, Spain; ${ }^{8}$ Hospital San Cecilio, Granada, Spain; ${ }^{9}$ Hospital Universitario Virgen de las Nieves, Granada, Spain; ${ }^{10} \mathrm{Hospital}$ Universitario Bellvitge, Hospitalet de Llobregat, Spain; ${ }^{11}$ Hospital Clínico Universitario De Santiago, Santiago de Compostela, Spain; ${ }^{12}$ Hospital la Paz, Madrid, Spain;

${ }^{13}$ Hospital Mutua Terrassa, Terrassa, Spain; ${ }^{14}$ Hospital Universitario Central de Asturias, Gijón, Spain; ${ }^{15}$ Sociedad Española de Medicina Interna, Madrid, Spain

Background: Anti-PM/Scl antibodies are associated to systemic sclerosis (SSc) but are not specific to SSc. The true prevalence of anti-PM/Scl antibodies in $\mathrm{SSc}$ is unknown, ranging from $2.5 \%$ to $12.5 \%$. An association between anti-PM/ $\mathrm{Scl}$ antibodies with muscular involvement, pulmonary fibrosis, calcinosis, and a relatively benign prognosis have been described.

Objectives: To compare the clinical manifestations and prognosis of SSc patients according the presence of anti-PM/Scl antibodies in the cohort of RESCLE (Spanish Scleroderma Registry).

Methods: From the Spanish Scleroderma Study Group database, we selected patients in whom anti-PM/Scl antibodies had been tested. We compared demographic features, clinical manifestations, laboratory characteristics, and survival data between patients according the anti-PM/Scl antibodies status.

Results: 72 out of 947 (7\%) patients tested positive for anti-PM/Scl antibodies. As presenting SSc manifestations, patients with anti-PM/Scl antibodies had higher prevalence of puffy fingers ( $11 \%$ versus $2 \% ; p=0.002)$ and arthralgias $(11 \%$ 
versus $4 \% ; p=0.03$ ), and lower prevalence of Raynaud's phenomenon (65\% versus $82 \%, p=0.002)$. Regarding cumulative manifestations, myositis ( $51 \%$ versus $15 \% ; p<0.001$ ), arthritis ( $43 \%$ versus $22 \% ; p=0.001$ ), and interstitial lung disease (ILD) $(60 \%$ versus $45 \%, \mathrm{p}=0.014)$ were more prevalent in patients with anti-PM/ $\mathrm{Scl}$ antibodies. In fact, those patients with anti- $\mathrm{Pm} / \mathrm{Scl}$ antibodies presented with FVC $(77.4 \% \pm 23.1 \%$ versus $85.8 \% \pm 23,1 \% ; p=0.006)$ and more severe ILD defined as FVC $<70 \%$ (41\% versus $24 \%$; $p=0.004)$. Death rate was similar in patients with and without $\mathrm{PM} / \mathrm{Scl}$ antibodies (18\% versus $17 \% ; \mathrm{p}=0.871)$. We did not find differences in terms of death rate nor in the causes of death (SSC and non-SSc related) according to the anti-PM/Scl antibodies profile. The 5- and 10 -years survival rates of patients with anti-PM/Scl antibodies were $91 \%$ and $82 \%$ respectively, without differences with those without these antibodies (93\% and $85 \%$, respectively)

Conclusion: In Spanish SSc patients, the presence of anti-PM/Scl antibodies confer a distinctive clinical profile. However, anti-PM/Scl antibodies do not play a role in the prognosis of these patients.

References:

[1] Stochmal A, Czuwara J, Trojanowska M, Rudnicka L. Antinuclear antibodies in systemic sclerosis: an update. Clin Rev Allergy Immunol 2020;58(1):40-51. doi: 10.1007/s12016-018-8718-8.

Acknowledgments: We gratefully acknowledge all investigators who are part of the RESCLE Registry. We also thank the RESCLE Registry Coordinating Centre, S\&H Medical Science Service, for their quality control data, logistic and administrative support and Prof. Salvador Ortiz, Universidad Autónoma de Madrid and Statistical Advisor S\&H Medical Science Service for the statistical analysis of the data presented in this paper.

Disclosure of InterestsNerea Iniesta-Arandia: None declared, Gerard Espinosa Speakers bureau: Glaxo-Smith-Kline, Janssen, Boehringer, Rovi, Alfredo Guillen del Castillo: None declared, Carles Tolosa Consultant of: Actelion pharmaceuticals, GSK, MSD., Gema Maria Lledó: None declared, Dolores Colunga Argüelles Consultant of: Actelion pharmaceuticals, GSK, MSD., Cristina GonzálezEchávarri: None declared, Luis Sáez-Comet: None declared, Norberto Ortego: None declared, Jose Antonio Vargas-Hitos: None declared, Manuel Rubio-Rivas: None declared, Mayka Freire: None declared, Juan José Rios: None declared, Monica Rodriguez-Carballeira: None declared, Luis Trapiella Martínez: None declared, Vicent Fonollosa Pla Speakers bureau: Actelion, Carmen Pilar Simeón-Aznar Consultant of: Actelion pharmaceuticals, GSK, MSD., on behalf of RESCLE Investigators, Autoimmune Diseases Study Group (GEAS): None declared

DOI: 10.1136/annrheumdis-2020-eular.3304

\section{SAT0317 HDL-CHOLESTEROL EFFLUX CAPACITY IS DOWNREGULATED IN PATIENTS WITH SYSTEMIC SCLEROSIS.}

1. Ferraz-Amaro ${ }^{1}$, D. F. Esmeralda ${ }^{1}$, V. Hernández-Hernández ${ }^{1}$, H. SánchezPérez $^{1}$, L. De Armas-Rillo² ${ }^{2}$ E. Armas González ${ }^{3}$, J. D. Machado ${ }^{4}$, F. DíazGonzález ${ }^{1}$. Division of Rheumatology, Hospital Universitario de Canarias, Tenerife, Spain, Santa Cruz de Tenerife, Spain; ${ }^{2}$ Universidad Europea de Canarias, Santa Cruz de Tenerife, Spain; ${ }^{3}$ Universidad de La Laguna, Departamento de Bioqui ímica, Microbiología, Biología Celular y Genética, La Laguna, Spain; ${ }^{4}$ Universidad de La Laguna, Santa Cruz de Tenerife, Spain

Background: It is well established that patients with systemic sclerosis (SS) show a disrupted lipid profile and an increased cardiovascular risk. Cholesterol efflux capacity (CEC) is the ability of high-density lipoprotein (HDL)-cholesterol to accept cholesterol from macrophages. CEC has been linked to cardiovascular events in the general population and to subclinical atherosclerosis in patients with rheumatoid arthritis and systemic lupus erythematosus.

Objectives: The main purpose of our study was to assess, for the first time, whether CEC is disrupted in patients with SS compared to controls. We also aimed to identify patients' characteristics that could explain such potential CEC disturbance.

Methods: Cross-sectional study that encompassed 188 individuals; 73 SS patients and 115 age- and sex-matched controls. CEC, using an in vitro assay, and lipoprotein serum concentrations were assessed in patients and controls. A multivariable analysis was performed to study the differences in CEC between patients and controls, and if SS-related data could explain CEC differences.

Results: CEC was downregulated in SS patients as compared to controls (beta coefficient $-6[95 \% \mathrm{Cl}-10--2] \%, p=0.002)$. This occurred independently of traditional cardiovascular risk factors, statin use or other variations in the lipid profile produced by the disease. Demographics and lipid profile were, in general, not related with CEC in both patients and controls. In this sense, only abdominal circumference showed a positive association with CEC in patients (beta coefficient 0.09 [95\% Cl $0.03-0.14], p=0.002$ ) but not in controls. Similarly, no traditional cardiovascular risk factors were related with CEC in both populations. Regarding lipid profile, no correlations were identified between the standard lipid profile molecules and CEC. Remarkably, the use of statins was not related to CEC in both patients and controls. Lastly, concerning SS related data, a negative association between $\mathrm{mRSS}$ and CEC was identified (beta coef. -1.08 [95\% Cl -2.03- -0.12$]$ $\%, p=0.028$ ).

Skin thickness through modified Rodnan (mRSS) was positively related to age and the presence of hypertension, but negatively associated with apolipoprotein $\mathrm{B}$, apo $\mathrm{B}: \mathrm{A} 1$ ratio, and $\mathrm{CEC}$ when univariate correlations were assessed (Table 4). When the relation of mRSS to these lipid-related molecules was adjusted for traditional CV risk factors, the statistical significance of mRSS with those molecules was maintained. Moreover, when the relation between mRSS and CEC was additionally adjusted for other lipid-related molecules, its significance was conserved (beta coef. $-1.35[95 \% \mathrm{Cl}-2.62--0.08]$ ) \% $\mathrm{p}=0.038$ )

Conclusion: CEC is downregulated in SS patients independently of other inflammation-related lipid profile modifications that occur in the disease. Skin thickness is independent and inversely associated with CEC in SS patients.

Disclosure of Interests: Iván Ferraz-Amaro Grant/research support from: Pfizer, Abbvie, Speakers bureau: Pfizer, Abbvie, MSD., delgado frias esmeralda Speakers bureau: Pfizer, Abbvie, MSD, Vanessa Hernández-Hernández Speakers bureau: Pfizer, Abbvie, MSD, Hiurma Sánchez-Pérez: None declared, Laura de Armas-Rillo: None declared, Estefania Armas González: None declared, Jose David Machado: None declared, Federico Díaz-González Grant/research support from: Abbvie, Pfizer, MSD, Speakers bureau: Abbvie, Pfizer, MSD

DOI: 10.1136/annrheumdis-2020-eular.620

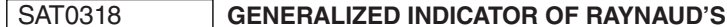 PHENOMENON EXPRESSION FOR EVALUATION OF CLINICAL EFFICACY OF PROSTANOID THERAPY}

I. Gaisin ${ }^{1}$, Z. Bagautdinova ${ }^{2}$, M. Glavatskikh ${ }^{3}$, N. Maximov ${ }^{1}$, R. Valeeva ${ }^{1}$, O. Desinova ${ }^{4}$, R. Shayakhmetova ${ }^{4} .{ }^{1}$ Izhevsk State Medical Academy, Izhevsk, Russian Federation; ${ }^{2}$ Clinical Diagnostic Centre of the Udmurt Republic, Izhevsk, Russian Federation; ${ }^{3}$ Udmurt State University, Izhevsk, Russian Federation; ${ }^{4}$ VA. Nasonova Research Institute of Rheumatology, Moscow, Russian Federation

Background: Raynaud's phenomenon (RP) secondary to rheumatic diseases (RD) can progress to irreversible tissue damage with digital ulceration, scarring and, rarely, gangrene requiring amputation ${ }^{1}$. Current medical treatments for RP are far from ideal: they are often either ineffective and/or poorly tolerated, thus a significant proportion of patients discontinue drug therapy ${ }^{2}$.

Objectives: To determine RP expression levels and to evaluate the long-term efficacy of iloprost and alprostadil in RP patients with RD.

Methods: Indicated therapy with intravenous iloprost $(n=10)$, alprostadil $(n=17)$ or their combinations $(n=13)$ was carried out for three years in patients with secondary RP in RD. Frequency of Raynaud's attacks, digital ulcers (DU) formation and pain intensity on visual analogue scale (VAS) were evaluated. A control group included 30 patients with RP in RD who did not receive prostanoid therapy. By factor analysis method a generalized index of $\mathrm{RP}$ expression was identified, on the basis of which levels of RP expression were determined.

Results: "RP expression" scale, revealed as an indicator of RP generalized manifestation, was an average value of two subscales: (1) consisted of 4 indices "DU", "digital pitting scars", "phalange amputation" and "frequency of Raynaud's attack", (2) included 3 indicators: "intensity of pain", "duration of illness", "whitening of fingers". Correlation of subscales showed their reliability $(r=0.294$, $p=0.053$ ). RP final expression (severity) was $1.51 \pm 0.86$. A low level of RP expression had values below 0.65 , a high level - over 2.37. At baseline, the high level of RP severity was defined in 16 (22.9\%) patients, medium - in 43 $(61.4 \%)$, low - in 11 (15.7\%).

RP treatment with iloprost was effective in the healing of DU in $100 \%$ of patients and led to decrease of RP expression generalized index from $2.25[1 ; 3]$ to 1.75 $[1 ; 2](p=0.012)$. Alprostadil therapy reduced pain intensity on VAS $(p<0.05)$ and numbness during Raynaud's attacks $(p<0.01)$ and decreased RP expression from $1[1 ; 2]$ to $1[0.5 ; 1.5](p=0.038)$. Patients on prostanoids combination had new DU and amputations; pain intensity reduced by $47 \%(p<0.05)$, RP expression generalized indicator did not change.

Conclusion: Based on RP clinical manifestations in RD patients, a generalized index of RP expression was identified and levels of RP severity were determined. Treatment with iloprost or alprostadil has significant effects on reducing the clinical manifestations of RP with a corresponding decrease in its severity. lloprost is indicated in patients with medium and high levels of RP expression index alprostadil - with medium and low index and non-effectiveness of calcium channel blockers. 